\title{
Tracheostomy in Head and Neck Cancers
}

\section{${ }^{1}$ Bachi T Hathiram, ${ }^{2}$ Reema Rai, ${ }^{3}$ Parag Watve, ${ }^{4}$ Vicky S Khattar}

${ }^{1}$ Professor and Head, Department of ENT and Head and Neck Surgery, TN Medical College and BYL Nair Charitable Hospital Mumbai, Maharashtra, India

${ }^{2,3}$ Resident, Department of ENT and Head and Neck Surgery, TN Medical College and BYL Nair Charitable Hospital, Mumbai Maharashtra, India

${ }^{4}$ Assistant Professor, Department of ENT and Head and Neck Surgery, TN Medical College and BYL Nair Charitable Hospital Mumbai, Maharashtra, India

Correspondence: Bachi T Hathiram, Professor and Head, Department of ENT and Head and Neck Surgery, College Building Ist Floor, TN Medical College and BYL Nair Charitable Hospital, Dr AL Nair Road, Mumbai Central, Mumbai-400061, Maharashtra India, e-mail: orlclinics@g.mail.com

\begin{abstract}
The term 'tracheostomy' or 'tracheotomy' refers to the procedure whereby a communication is made between the anterior wall of the trachea and the exterior which bypasses the upper air passages. It results in the formation of a fistulous tract between the anterior aspect of the neck and the trachea allowing air to enter the lower respiratory passages without passing through the nose, pharynx and larynx.

It is performed at all ages, however, there are significant differences in the procedure when performed in adults and children.

'Laryngotomy' or 'cricothyrotomy' is the emergency operation where the airway is opened through the cricothyroid membrane in case of acute respiratory obstruction where there is no time for tracheostomy or inavailability of a competent surgeon to perform the tracheostomy. It is a relatively simple procedure and life-saving.
\end{abstract}

Keywords: Tracheostomy, Head and neck cancer.

\section{HISTORICAL REVIEW}

Historical accounts of this operation vie with each other in plumbing the depths of antiquity for plausible evidence..$^{1-4}$

Since the renaissance, this life-saving operation has been better described, and certain trends have become apparent. The first successful tracheostomy in a child was reported by Caron, a French surgeon in 1766 who removed an inhaled bean from a 7-year-old boy. The other common indications for this operation were 'croup' or 'diphtheria'. In the nineteenth century, tracheostomy was widely performed for diphtheria in children to relieve them of the upper airway obstruction often seen in this disease. The use of tracheostomy for the removal of bronchial secretions was first described by Galloway in patients of bulbar poliomyelitis.

However, Chevalier Jackson is credited with the first clear open surgical (OS) description in 1909, and Ciaglia is credited with the first percutaneous dilatational tracheostomy (PDT) in $1985 .{ }^{6,15}$ A procedure that previously required an Operating Room (OR) is now commonly performed in the Intensive Care Unit (ICU) ${ }^{16,17}$ Knowledge of tracheostomy is therefore still very important for surgeons; however, it is equally important for those responsible for patient care in the ICU.

\section{CLASSIFICATION}

Tracheostomy can be classified into:
I. a. Emergency
b. Elective/Planned
II. a. Temporary
b. Permanent
III.a. High
b. Mid
c. Low.

Emergency tracheostomy is performed in a case of acute respiratory distress. Occasionally, an endotracheal tube can be passed in an emergency to tide over the crisis and if the tube is required for more than 48 hours, a tracheostomy is performed to prevent laryngeal damage (vocal cord granulomas, etc.) However, intubation may not be possible in every case of acute respiratory distress, e.g. in case of large malignancy of larynx, laryngeal papillomatosis, etc. Elective/Planned tracheostomy is performed whenever respiratory distress or upper respiratory obstruction is anticipated, e.g. prior to certain surgical procedures such as hemimandibulectomy, hemiglossectomy, etc. Temporary tracheostomy is performed, if the causative factor can be removed or cured, e.g. laryngeal edema, trauma, foreign body larynx, etc. Permanent tracheostomy is performed 
when the causative factor cannot be treated or removed, e.g. crush injuries of larynx, laryngectomy operation, etc. High tracheostomy is performed rarely, only in a dire emergency. It is performed at level of 1st or 2nd tracheal rings and has a very high-risk of tracheal or subglottic (if cricoid cartilage is damaged) stenosis at a later date. Mid tracheostomy is performed most commonly and it is at the level of 3rd or 4th tracheal rings. Low tracheostomy is performed below level of 4th tracheal ring and is rarely done, e.g. in cases of tracheal stenosis, etc.

\section{INDICATIONS}

Common indications of tracheostomy seen in practice:

Foreign body in the larynx, Head injury resulting in unconsciousness,

Malignancy of the larynx, Laryngeal diphtheria,

Tetanus, Acute laryngotracheobronchitis.

Indications of tracheostomy:

In cases of upper airway obstruction due to the following causes

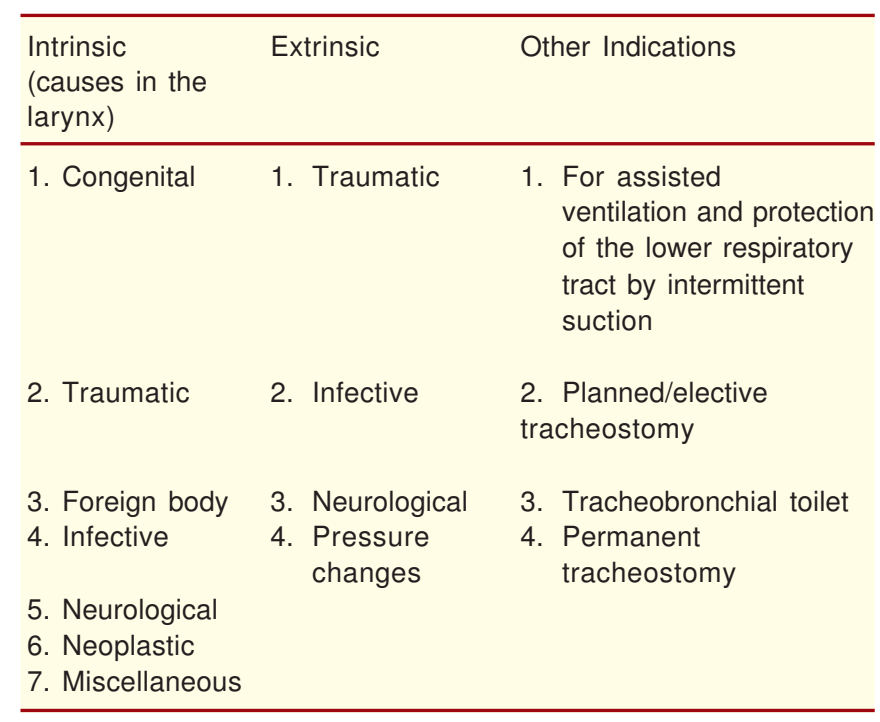

\section{Indications Due to Pathology of the Larynx (Intrinsic)}

\section{Congenital:}

- Laryngeal web.

- Tracheoesophageal fistula.

- Laryngeal stenosis.

Traumatic:

- Inhalation of fumes.

- Swallowing of corrosives.

\section{Foreign body:}

- Impacted foreign body in the larynx or the trachea requires a tracheostomy to:

- By-pass the airway obstruction,

- Prevent further aspiration of the foreign body in the lower respiratory tract.

\section{Infective:}

- Laryngeal diphtheria.

- Acute epiglottitis.

- Acute laryngotracheobronchitis.

Neurological:

- Bilateral abductor cord palsy.

- Myasthenia gravis.

- Tetanus.

- Bulbar poliomyelitis.

Neoplastic:

- Benign Laryngeal papillomatosis.

- Malignant Carcinoma of oropharynx, Carcinoma of larynx.

\section{Extrinsic Indications}

\section{Traumatic:}

- Cut-throat injury.

- Crush injury to larynx.

- Strangulation injury.

\section{Infective:}

- Ludwig's angina.

- Retropharyngeal abscess.

- Parapharyngeal abscess.

Neurological:

- Carcinoma of esophagus involving the recurrent laryngeal nerve.

- Carcinoma of thyroid involving the recurrent laryngeal nerve.

- Metastatic mediastinal lymph nodes involving the recurrent laryngeal nerve.

- Post-thyroidectomy trauma to both recurrent laryngeal nerves.

\section{Pressure changes:}

- Pressure compression of the trachea due to malignant thyroid tumor.

- Tumor of the neck causing pressure on larynx or trachea.

\section{Other Indications}

For assisted ventilation and protection of lower airway by intermittent suction:

- Unconscious or comatose patient due to head injury, encephalitis, cerebrovascular accident, etc. 
- Respiratory center depression due to bulbar palsy, barbiturate poisoning, etc.

- Lesions affecting the anterior horn cells and nerves controlling the muscles for respiration such as poliomyelitis, polyneuritis, myasthenia gravis, etc.

- Lesions affecting the myoneural junction, such as tetanus.

- Lesions affecting the chest wall such as multiple rib feactures, flail chest, etc.

- Lung pathology like fibrosis, collapse, emphysema, etc.

- Grave injury to face, neck and chest, e.g. burns.

Planned/Elective Tracheostomy: Usually done prior to major surgery of the head and neck to avoid aspiration after surgery and to maintain an airway, e.g. Prior to operations such as laryngofissure, hemiglossectomy, mandibulectomy, drainage of retropharyngeal abscess and operations for faciomaxillary injuries.

Tracheobronchial toilet: For example, removal of secretions from the lower respiratory tract in diseases such as COPD (emphysema, bronchiectasis).

\section{CONTRAINDICATIONS}

There is no such contraindication to performing a tracheostomy when required as it is a life-saving procedure. However, only in cases of planned/elective tracheostomy, relative contraindications such as diabetes mellitus, hypertension, bleeding disorder, etc. may be controlled prior to performing the tracheostomy.

\section{Types of Tracheostomy Tubes}

Metallic (Chevalier Jackson tracheostomy tube): It is made of German Silver. Has 3 parts-Inner tube, Outer tube, and Obturator/guide/pilot. The inner tube is longer than the outer tube by $2-3 \mathrm{~mm}$ so that when the tube gets blocked, the blockage is cleared by removing the inner tube. It is fixed to the outer-tube by a locking mechanism and the outertube is fixed by a linen tape passed through the holes of the shield attached to the proximal end of the outer tube and tied around the neck. The obturator has a blunt olive tip and is passed through the outer-tube during insertion of the tracheostomy tube into the trachea so as to make the distal end atraumatic. Once, the outer tube is in position, the obturator is removed and the inner tube is introduced and locked in position.

Available in sizes from No. 8 to 44 and only in even numbers, i.e. $8,10,12$, etc.
Size used commonly for: Normal adult male - No. 34 or 36, Normal adult female - No. 32 or 34 .

Portex tracheostomy tube: It is made of portex and has a blue line running through the entire extent of the tube which is impregnated with barium salt so as to make the tube radio-opaque. It has 2 parts-The Tube, and the Obturator. No separate inner and outer tubes, since portex is not as rigid as metal, the walls of the tube are thick so as to maintain the patency and shape of the tube. The walls are 0.7 to $1.2 \mathrm{~mm}$ thick. It is available from sizes 3 to 10 in increments of 0.5 , i.e. $3,3.5,4,4.5$, etc. The size used commonly for a-Normal adult male - No. 9 or 10; Normal adult female No. 8 or 9. All tubes of size No. 5 and below are without a cuff and tubes No. 5.5 or above are available in 2 typeswith or without cuff. The cuff is present at the distal portion of the tube and requires 2 to $3 \mathrm{cc}$ of air for inflation. The pressure is indicated roughly by a small pilot bag attached to the flange/shield of the tube.

Advantages of cuff: The cuff helps to make an airtight seal with the lower respiratory tract, i.e. air cannot escape between the tube and the lumen of the trachea due to the cuff hence a cuffed tube can be used for: Anesthesia, patients on ventilator, and Intermittent positive pressure respiration.

The portex tracheostomy tube is kept in position by means of a linen tape passed through the shield/flange attached to it's proximal end and tied around the neck.

Salpekar's double-cuffed tracheostomy tube: It has 2 cuffs at the distal portion. This is useful in cases where the cuff has to be kept inflated for a prolonged period continuously, e.g. comatose patient on ventilator. The advantage of two cuffs is that, one cuff is inflated for a while and the other is kept deflated alternately so as to avoid pressure continuously at one site on the trachea and thus avoid pressure necrosis.

Fuller's bivalved tracheostomy tube: The tube consists of an inner tube and an outer tube. The outer tube is bivalved. The two cusps of the outer tube are compressed and the tube is easily inserted into the tracheal lumen without either a pilot or tracheal dilator. The inner tube is then inserted. The inner tube has an opening on the posterosuperior wall which helps in decannulation, i.e. patient can breathe through the upper respiratory tract on blocking the tracheostomy opening. This also helps in speech when the tube is plugged and may be used when tracheostomy is performed, albeit rarely for the management of obstructive sleep apnea where the patient plugs the tube during the day and can speak, and 
unplugs it at night to prevent apneic spells and ensure a sound sleep.

Durham's tube: This has an adjustable shield/flange and hence is very useful in patients with a short fat neck.

Radcliff tube: This is a right angled tube and is used in patients with a short fat neck.

Also, tracheostomy tubes can be attached to a speaking valve, which is a one way Duck-Bill type of valve and helps the patient to speak. Valves available include - Rusch valve, Disanti valve and Tucker valve.

\section{PROCEDURE}

\section{Preprocedure preparation:}

- Written informed consent of patient and/or relative. In the event of an extreme emergency, this need not be done as tracheostomy is life-saving.

- 0.5 cc Inj Tetanus Toxoid intramuscularly.

- $0.6 \mathrm{mg}$ Inj Atropine sulfate intramuscularly half-hour before the procedure.

- Inj. Lignocaine test dose.

- Tracheostomy tube, instruments, suction machine and good illumination should be checked, prior to the procedure.

- Strict aseptic precautions are taken.

Anesthesia: Can be performed under general anesthesia with endotracheal intubation or under local infiltration anesthesia. The former is preferred in planned/elective tracheostomy and the latter is preferred in emergency tracheostomy.

Position: Patient is in the supine position with full extension of the neck and head. This is obtained by placing a sandbag or pillow under the patient's shoulders. Neck and head must be held in the midline. This position brings the cervical trachea forward in the neck and more tracheal rings are accessible in the neck.

Steps: A midline vertical neck incision from lower border of cricoid cartilage to the suprasternal notch is taken using a No. 11 surgical blade on a Bard-Parker handle. Horizontal neck incision midway between the lower border of cricoid cartilage and suprasternal notch is used rarely only in cases of elective tracheostomy. The neck incision is deepened in the midline cutting the superficial and deep cervical fascia and subcutaneous fat. The anterior jugular vein or transverse cervical veins may be encountered. Dissection is carried out with a curved mosquito hemostat, strictly in the midline.
The midline raphe between the two sets of strap muscles on either side of the neck is encountered, dissected and strap muscles are retracted on either side by right-angled retractors or double-hook retractors. The thyroid isthmus is now exposed and it is retracted upwards with a single hook retractor to expose the 2 nd and 3rd tracheal rings. Some surgeons prefer to clamp and cut the thyroid isthmus but, this may cause bleeding and loss of precious time. The pretracheal fascia is incised with the surgical blade and dissected away from the tracheal rings with the curved mosquito hemostat. The tracheal rings are thus exposed and this is confirmed by palpation of the rings and aspiration of air from the trachea with a needle and syringe filled with $1 \mathrm{cc}$ of $4 \%$ Lignocaine solution. Once air-bubbles are seen in the syringe, $0.5 \mathrm{ml}$ of $4 \%$ Lignocaine is instilled into the tracheal lumen to anesthetise the mucosa and also prevent a vaso-vagal attack. With the surgical knife an incision is made over the 2 nd and 3rd tracheal rings on the anterior tracheal wall. There are various incisions which can be used. The cruciate or circular incisions are commonly used. However, the horizontal incision does not pass through cartilage and hence helps to minimize cartilage necrosis. This is preferred in children. The tracheal opening is dilated with a tracheal dilator and the tracheostomy tube is introduced with the obturator/guide in position. Following insertion, the obturator is removed and the presence of the tube in the tracheal lumen in confirmed by:

- Air-blast felt on the back of the hand,

- Movement of a wisp of cotton placed near the opening of the tube,

- Auscultation of chest for bilaterally equal air entry, and

- If patient is on ventilator, then auscultation of chest after attachment of ventilator will confirm presence of tube in correct position.

In case of metallic tube, the inner tube is placed into the outer tube and fixed in place with the locking mechanism. The tracheostomy tube is fixed in position by tying it to the neck with the help of linen tapes passed through the holes in the shield/flange. In case of cuffed portex tubes, the cuff is now inflated with 2-3 cc of air. A dressing is given in the form of a few sterile gauze pieces cut and inserted between the shield of the tube and skin.

A plain radiograph of the neck and chest should be taken immediately after the procedure to confirm the correct placement of the tube as well as for documentation and medicolegal purposes, should the issue arises at some later date. 


\section{POSTOPERATIVE CARE / TRACHEOSTOMY CARE}

The following should be constantly by the patient's bedside: A set of tracheostomy instruments and a tracheostomy tube of the same size and type; a bell to call the nurse and a piece of paper with pen for communication, if patient is literate; suction apparatus; and oxygen.

Suction through the tube every half hourly and instillation of $0.5 \mathrm{ml}$ of sodium bicarbonate into the tube prior to each suction. This helps to liquefy the crusts and aids in suction of secretions. It is important to remember that the suction catheter should be inserted without suction and negative pressure is exerted when the catheter is withdrawn. Also, the size of the catheter should be less than half the inner diameter of the tracheostomy tube. If this is not kept in mind, it may result in hypoxia or lung collapse.

In case of metallic tube, the inner tube should be removed, cleaned, sterilized (boiled) and replaced every 4 hours or earlier if required.

In case of a cuffed portex tube, the cuff needs to be deflated for 10 minutes every two hours to prevent pressure necrosis of the tracheal rings.

A single moist gauze piece is placed at all times over the opening of the tracheostomy tube to help in humidification of air entering the lower respiratory tract and prevent entry of foreign bodies like insects, etc.

The tracheostomy dressing is changed every day using sterile gauze pieces.

Steam inhalations twice or thrice a day to prevent excessive drying of secretions and regular daily physiotherapy is given to avoid lower respiratory tract infection.

Change of tracheostomy tube is to be avoided for the first 48 hours following a tracheostomy since by this time a tract forms making reinsertion of the tube easier. However, a tracheal dilator should always be used during change of tracheostomy tube.

\section{DECANNULATION}

It is important to reduce the size of the tube prior to decannulation as there should be sufficient 'breathing space' between the wall of the trachea and the wall of the tube for the patient to breathe when the tracheostomy tube is corked. The tube is initially corked for a few hours. The interval is then gradually lengthened so that the patient can tolerate the tube being corked for 12 hours in the daytime. Following this, the patient's tube is corked for the entire night and only when this is well-tolerated without any further need for tracheal suction, the patient may be decannulated. A dressing is given for 3 to 5 days.

A surgical decannulation may be performed where the tracheostomy tract is excised and the tracheal stoma is directly visualized and surgically closed over an endotracheal tube which is removed after 2 to 4 hours. Prior to closure, the tracheal lumen is visualized endoscopically to rule out any intraluminal cause of obstruction.

\section{SEQUELAE}

The problems faced by the patient following a tracheostomy are:

- Inability to speak: This is due to the larynx being bypassed,

- Difficulty in taking a shower or washing his head,

- Inability to swim,

- Inability to lift heavy weights,

- Difficulty in straining during micturition, constipation or child-birth. This is due to the fact that, there is a difficulty in increasing the intra-abdominal pressure required for these activities as the thorax cannot be fixed,

- Anosmia as the nose which contains the olfactory nerve endings is bypassed,

- $50 \%$ of the dead space is eliminated by a tracheostomy, thus reducing airway resistance and making breathing easier.

\section{SPECIAL CONSIDERATIONS FOR PERFORMING A TRACHEOSTOMY IN A PATIENT WITH HEAD AND NECK MALIGNANCY}

The tracheostomy performed in a patient with head and neck malignancy can either be performed as an emergency, when the patient presents in stridor; or electively planned, prior to surgery where endotracheal intubation is not feasible or where airway obstruction is anticipated in the postoperative period. It is important to remember that a tracheostomy should be avoided if possible in a patient who requires laryngectomy since, this would enable us to have a good length of disease-free trachea for the subsequent tracheostoma and would also prevent peristomal recurrence which frequently complicates a tracheostomy performed preoperatively in these patients. If a tracheostomy is performed in a patient who requires a laryngectomy, it would be advisable to perform the laryngectomy within 24 to 48 hours of the tracheostomy to prevent peristomal 
recurrence. In patients with stridor, where a laryngectomy is anticipated for the treatment of the disease, a high tracheostomy through the first or second tracheal rings is preferably performed since this leaves the healthy tracheal rings for formation of the tracheostoma after surgery. However, there is a school of thought which still prefers an emergency laryngectomy to performing a preoperative tracheostomy. Prior to performing an emergency tracheostomy in a previously unseen stridulous patient, it would be a good idea to get a digital radiograph of the neck done to rule out invasion of the trachea by the growth, since such a finding during the procedure could be disasterous.

A difficult situation would be the involvement of the anterior neck by the malignancy, when the surgeon has to use his skill and experience to deal with the situation. Such tracheostomies are best performed in the operation theatre with all facilities such as suction, cautery and all resuscitative equipment available at hand should the need arise. In the event of hemorrhage during the procedure from the growth, it is better to apply local pressure and put the tracheostomy tube in situ thus, first securing the airway and then, dealing with hemostasis. An elective preoperative tracheostomy is comfortably performed in the operation theatre and care is taken not to damage the tracheal rings by using the intercartilagenous inscision. Tracheostomy in an irradiated neck poses a challenge since all tissues are fibrosed and there are no tissue planes. Dissection is difficult and may require a scalpel or cautery to reach the trachea.

\section{COMPLICATIONS}

As with any surgical procedure, complications are known to occur during this life-saving procedure as well. Depending on the time of occurance, they may be divided into; immediate-during or immediately after the procedure, delayed -24 hours after the procedure and late—occurring after a few weeks.

\section{Immediate Complications}

Hemorrhage: Primary hemorrhage may occur during the procedure due to injury to the following:

- Thyroid isthmus,

- Transverse cervical vessels,

- Strap muscles,

- High innominate artery especially in children,

- Carotid artery if dissection is carried out more laterally usually may occur in children,
- Jugular vein - may occur in children if dissection is carried out more laterally.

This can be avoided by meticulous dissection in the midline under good illumination.

Injury to the esophagus: Resulting in tracheoesophageal fistula.

Injury to the dome of pleura: Common in children.

Injury to recurrent laryngeal nerve.

Injury to cricoid cartilage: Common in high tracheostomy. Apnea: Due to central respiratory failure - usually may occur in a patient of prolonged dyspnea. As soon as the trachea is incised, there is a carbondioxide wash-out. In such patients hypoxia is the stimulus for the respiratory center. This stimulus is lost suddenly when $\mathrm{CO}_{2}$ wash-out occurs and $\mathrm{O}_{2}$ levels in the body rise, this results in apnea. This can be treated by administration of carbogen (95\% oxygen and 5\% carbondioxide).

Vasovagal attack: Due to vagal stimulation. This is prevented by instilling $0.5 \mathrm{cc}$ of $4 \%$ lignocaine into the tracheal lumen before incising the trachea.

Aspiration and lung collapse.

Aerophagia: May occur in infants.

\section{Delayed Complications}

Reactionary hemorrhage: Occurs within 48 hours.

Secondary hemorrhage: Occurs on the 5th to 8th day usually but can occur at anytime after 48 hours. Antibiotics should be administered and the wound should be explored to find the bleeding vessel which may have undergone necrosis. Surgical emphysema: Occurs due to air which leaks out from around the tracheostomy tube through the tracheal lumen but cannot escape out due to tight suturing of the skin incision. Hence, this exhaled air is forced into the subcutaneous tissues of the neck and is characterized by crackling feel on palpation at the root of the neck followed by bloating up of the skin. It is prevented by taking care not to suture the skin wound too tightly around the tracheostomy tube. It can be treated by releasing the tight sutures. If untreated, it may involve the subcutaneous tissues of the entire body and require multiple punctures or small incisions over the skin. This can occur only in a non-cuffed portex or metal tube.

Mediastinal emphysema: Occurs due to escape of air from the tracheal lumen around the tracheostomy tube under the pretracheal fascia into the pretracheal space which communicates inferiorly with the superior mediastinum. This is prevented by incising the pretracheal fascia adequately 
and retracting it away from the site of the tracheal incision. This air leak can occur only in a metallic or non-cuffed portex tube.

Delayed tracheoesophageal fistula: This occurs due to pressure necrosis of the tracheal wall if the cuff is not deflated at regular intervals.

Tracheobronchitis: May occur as the lower respiratory tract is exposed to the exterior or if septic precautions are not taken during change of dressing or suction of trachea.

Pneumothorax and pyopneumothorax.

Dysphagia: This occurs due to the pressure of the cuff on the trachea in case of a cuffed portex tube, and due to a lack of positive subglottic pressure during deglutition.

Blocking of the tube: Due to drying of secretions and crust formation.

\section{Late Complications}

Tracheomalacia: Occurs if a large area of the tracheal wall has been removed during tracheostomy or if a cuffed tube has been used for prolonged period without deflation of cuff resulting in pressure necrosis of tracheal rings.

Laryngeal stenosis: In case of damage to cricoid cartilage in a high tracheostomy.

Tracheal stenosis: Tracheal stenosis, an abnormal narrowing of the tracheal lumen, most commonly occurs at the level of the stoma or above the stoma (suprastomal) but below the vocal cords (subglottic). ${ }^{10-12}$ Tracheal stenosis may also occur at the site of tracheostomy tube cuff or at the site of the tube's distal tip. Stomal stenosis develops secondary to bacterial infection and chondritis, which conspire to weaken the anterior and lateral tracheal walls. Stomal granulation tissue frequently develops, and nearly all patients have some degree of tracheal narrowing at the site of the tracheostoma. In case of prolonged use of cuffed tube, cartilage necrosis at the site of the cuff may result in stenosis after decannulation. The incidence of cuff (infrastomal) stenosis has fallen 10-fold with the change from low-volume highpressure to high-volume low-pressure cuffs. ${ }^{13,14}$ Unfortunately, overinflation of a high-volume low-pressure cuff can lead to ischemic airway injury.

Persistent tracheocutaneous fistula even after decannulation: This may require surgical closure.

Depressed scar: May occur at times following healing of the tracheostomy skin wound.

\section{Tracheoinnominate-artery erosion:}

One of the most feared complications of tracheostomy is the development of a tracheoinnominate-artery fistula. ${ }^{10}$
Risk factors for the development of tracheoinnominate fistula include excessive movement of the tracheostomy, high pressure (or overinflated) cuff, or a tube that has been placed too low. The innominate artery lies adjacent to the trachea and crosses that structure at approximately the 9th tracheal ring. If the tracheostomy tube is placed too low, below the 3rd tracheal ring, the inferior concave surface of the cannula may erode into the artery. Alternatively, an overinflated tracheostomy cuff balloon or the tip of the tracheostomy tube can severely damage the tracheal mucosa, leading to necrosis and eventual erosion into the innominate artery. This complication occurs in less than $1 \%$ of all patients undergoing tracheostomy. The vast majority of cases (approximately 75\%) will occur within 3 to 4 weeks of tracheostomy placement. The mortality rate approaches $100 \%$, even when surgical intervention is undertaken.

Difficulty in decannulation: This may occur due to the following reasons:

- Psychological dependence on the tracheostomy tube. This is common in children and occurs because the patient gets accustomed to breathing without resistance as the upper respiratory tract (which gives a natural resistance to air flow) is bypassed. Hence, when decannulated, the patient gets breathless even though the pathology which caused dyspnea is cured.

- Inadequate treatment of cause of dyspnea which results in persistence of the obstruction and hence when decannulated, the patient may get breathless.

- Presence of granulations in the tracheal lumen at site of tracheostomy.

- Tracheomalacia.

- Hence, in such a case, one should first ascertain that the cause of dyspnea is treated completely and absent. In case of psychological dependence the following is done.

- Decannulate as soon as cure is achieved.

- Partial blocking of the tube and gradual decannulation over a period of 2 to 3 days.

\section{A FEW WORDS OF CAUTION WHEN PERFORMING A TRACHEOSTOMY IN INFANTS AND CHILDREN}

A tracheostomy should be avoided if endotracheal intubation can be performed to maintain airway in an acute situation. The larynx is higher in the neck. The trachea is softer and relatively deeper beneath the skin. Landmarks are less easily palpable. A low tracheostomy should be avoided at all cost. The apical pleura, great vessels of the neck and the 
innominate artery may be easily damaged in a child. Avoid excision of tracheal cartilage during opening the trachea. A vertical incision or a horizontal intercartilagenous incision is preferred. Avoid too deep an incision as the tracheal lumen is narrow and the incision may go through the esophageal wall. Be prepared for decannulation problems as psychological dependence is more common in children.

\section{PERCUTANEOUS TRACHEOSTOMY}

In 1969 the percutaneous tracheal dilatation to overcome airway obstruction in the critical care setting was first described. ${ }^{5}$ It is a procedure not really suitable for the management of acute airway obstruction but, used as an elective procedure. A percutaneous tracheostomy is defined as the insertion of a tracheostomy tube without direct visualization of the anterior tracheal wall, using minimal dissection through the smallest incision possible.

Several proprietary techniques exist, but all employ a modified Seldinger Technique. ${ }^{18}$ There are two basic methods of percutaneous tracheostomy: the Griggs' technique and the Ciaglia's technique. The site of insertion for both is between the second and third tracheal rings. A Seldinger guide wire is inserted into the tracheal lumen to guide the tube into position after which it is removed. ${ }^{6,7}$

Concomitant bronchoscopy adds a "tracheal view" that helps reposition the endotracheal tube (ETT) above the incision and helps to visualize needle placement and subsequent stomal dilatation. Bronchoscopy can also reduce posterior tracheal wall injury, confirm tube placement and help airway toilet. It is therefore strongly recommended. ${ }^{19-21}$

A modification of the Ciagla's technique has gained popularity nowadays. ${ }^{8}$ The blue rhino allows full dilation by means of a hydrophilically coated, curved dilator. This is a single-step procedure and it greatly reduces the risk of posterior tracheal wall injury and intraoperative bleeding. ${ }^{9}$

\section{CRICOTHYROIDOTOMY}

This is also known as a "mini-tracheostomy". It is an emergency life-saving procedure performed for acute airway obstruction where there is no time for a conventional tracheostomy. A mid-line incision is made in the relative avascular cricothyroid membrane. A small diameter tube is then inserted over a guide-wire through a percutaneous approach using a dilator. It is only suitable as an emergency life-saving measure and soon a conventional tracheostomy is required.
It has a high incidence of subglottic stenosis and voice dysfunction. It is not recommended in children. Also, it cannot be performed in a subglottic pathology.

\section{REFERENCES}

1. Goodall EW. The story of tracheostomy. British J Childhood Dis 1934;31:167-76.

2. Salmon LFW. Tracheostomy: The evolution of an operation. Guy's Hospital Gazette 1957;71:233-42.

3. Nelson TG. Tracheostomy: A clinical and Experimental study. Baltimore: 1958; Williams and Wilkins.

4. Frost EAM. Tracing the Tracheostomy. Ann Otol Rhinol. Laryngol 1976; 85:618-24.

5. Bouderka MA, Fakhir B, Bouaggad A, Hmamouchi B, Hamoudi D, Harti A. Early tracheostomy versus prolonged endotracheal intubation in severe head injury. J Trauma 2004;57(2):251-54.

6. Ciaglia P, Firsching R, Syniec C. Elective percutaneous dilational tracheostomy: A new simple bedside procedure; preliminary report. Chest 1985;87:715-19.

7. Dulguerov P, Gysin C, Perneger TV, Chevrolet JC. Percutaneous or surgical tracheostomy: A meta-analysis. Crit Care Med 1999;27:1617-25.

8. Kost KM. Endoscopic percutaneous dilatational tracheostomy: A Prospective evaluation of 500 consecutive cases. Laryngoscope 2005;115:1-4.

9. Toye FJ. Weinstein, JD. A percutaneous tracheostomy device. Surgery 1969;65:384-89.

10. Sue RD, Susanto I. Long-term complications of artificial airways.Clin Chest Med 2003;24(3):457-71.

11. Stauffer JL, Olson DE, Petty TL. Complications and consequences of endotracheal intubation and tracheotomy: A prospective study of 150 critically ill adult patients. Am J Med 1981;70(1):65-76.

12. Streitz JM Jr, Shapshay SM. Airway injury after tracheotomy and endotracheal intubation. Surg Clin North Am 1991;71(6):1211-30.

13. Lewis FR Jr, Schiobohm RM, Thomas AN. Prevention of complications from prolonged tracheal intubation. Am J Surg 1978;135(3):452-57.

14. Leigh JM, Maynard JP. Pressure on the tracheal mucosa from cuffed tubes. Br Med J 1979;1(6172):1173-74.

15. Jackson C. Tracheotomy. Laryngoscope 1909;19:285-90.

16. Delaney A, Bagshaw SM, Nalos M. Percutaneous dilatational tracheostomy versus surgical tracheostomy in critically ill patients: A systematic review and meta-analysis. Crit Care. 2006; 10:R55.

17. Griffiths J, Barber VS, Morgan L, et al. Systematic review and meta-analysis of studies of the timing of tracheostomy in adult patients undergoing artificial ventilation. Br Med J 2005;330:1243.

18. Seldinger SI. Catheter replacement of the needle in percutaneous arteriography: A new technique. Acta Radiol 1953;39:368-76.

19. Silvester W, Goldsmith D, Uchino S, et al. Percutaneous versus surgical tracheostomy: A randomized controlled study with longterm follow-up. Crit Care Med 2006;34:2145-52.

20. deBoisblanc BP. Percutaneous dilational tracheostomy techniques. Clin Chest Med 2003;24:399-407.

21. De Leyn P, Bedert L, Delcroix M, et al. Tracheotomy: Clinical review and guidelines. Eur J Cardiothorac Surg 2007;32:412-21. 